\title{
Influence of insonification angle on echogenicity of B-mode images of atherosclerotic
} plaque in vitro

Wilhjelm, Jens E.; Jespersen, Søren Kragh; Hansen, J. U.; Nyssen, N.I.H.

Published in:

I E E E International Ultrasonics Symposium. Proceedings

Link to article, DOI:

10.1109/ULTSYM.1998.765250

Publication date:

1998

Document Version

Publisher's PDF, also known as Version of record

Link back to DTU Orbit

Citation (APA):

Wilhjelm, J. E., Jespersen, S. K., Hansen, J. U., \& Nyssen, N. I. H. (1998). Influence of insonification angle on echogenicity of B-mode images of atherosclerotic plaque in vitro. I E E E International Ultrasonics Symposium. Proceedings, 2, 1599 - 1602. https://doi.org/10.1109/ULTSYM.1998.765250

\section{General rights}

Copyright and moral rights for the publications made accessible in the public portal are retained by the authors and/or other copyright owners and it is a condition of accessing publications that users recognise and abide by the legal requirements associated with these rights.

- Users may download and print one copy of any publication from the public portal for the purpose of private study or research.

- You may not further distribute the material or use it for any profit-making activity or commercial gain

- You may freely distribute the URL identifying the publication in the public portal 


\title{
Influence of Insonification Angle on Echogenicity of B-Mode Images of Atherosclerotic Plaque in vitro
}

\author{
J. E. WILHJELM ${ }^{1}$, S. K. JESPERSEN ${ }^{2}$, J. U. HANSEN ${ }^{1}$, N. I. H. NYSSEN ${ }^{1}$ \\ Center for Arteriosclerosis Detection with Ultrasound (CADUS) \\ E-mail: wilhjelm@it.dtu.dk. Homepage: http://www.it.dtu.dk/ wilhjelm/cadus.html \\ ${ }^{1}$ Dept. of Information Technol., Tech. Univ. of Denmark, Bldg. 344, DK-2800 Lyngby, Denmark. \\ ${ }^{2}$ B-K Medical A/S, Sandtoften 9, DK-2820 Gentofte, Denmark.
}

\begin{abstract}
A newly developed (off-line) spatial compound scanner was used to scan formalin-fixed atherosclerotic carotid plaques. Forty-eight B-mode images were recorded using 7 insonification angles.

All calculations were done on the envelope-detected image data. The mean amplitude level (MAL) in (relative) volts was calculated for the plaque region in each image.

The standard deviation over the $48 \mathrm{MAL}$ values were for each of the 7 angles between $0.12 \mathrm{~V}$ and $0.18 \mathrm{~V}$. For each scan plane, the standard deviation was also calculated over the 7 images. The mean and standard deviation of these 48 numbers were $0.07 \mathrm{~V}$ and $0.04 \mathrm{~V}$, respectively. Thus based on these data, the variation from scan plane to scan plane is normally larger than the variation over angle for a single scan plane.
\end{abstract}

\section{Introduction}

For the purpose of assessing the risk of stroke, several in vivo studies ${ }^{[1,2,3]}$ have investigated the relation between the relative amount of soft materials in atherosclerotic plaque removed by surgery and the mean echogenicity of in vivo ultrasound images of these plaques. The correlation is quite weak, and one of the reasons might be the variation in echogenicity with insonification angle for non-soft tissues.

In an attempt to investigate this, a newly developed spatial compound scanner was used to scan formalin-fixed atherosclerotic plaque removed by surgery (endarterectomy). The mean amplitude level was calculated for the plaque region in all images and the variation of this level over scan plane and angle was calculated and analyzed.

\section{2 . Ultrasound System}

The ultrasound system consisted of a 192-element linear array transducer with low pitch connected to a 64-channel (off-line) ultrasound system, as depicted in the lower part of Figure 1. The system was controlled by a real-time scan controller and operated from a workstation. Additional description of the system can be found elsewhere in this proceeding. ${ }^{[4]}$ The system operates by emitting beams in a specified direction (below $\left.30^{\circ}\right)^{[4]}$ from an aperture of 64 elements and then recording the received single-element signal, each element at a time, from the same 64 element aperture. Specifically, each of the 64 single-element signals are preamplified by its own preamplifier and then one signal is selected with a switch, amplified with Time Gain Compensation (TGC) and eventually digitized with a resolution of 12 bits at up to $60 \mathrm{MHz}$ sampling rate. All signal processing is performed in software, including the dynamic focusing at receive. Subsequent signal processing consists of bandpass filtering, envelope detection, scan conversion, logarithmization, scaling and display.

The implementation of the signal processing in software has the advantage that very high quality ultrasound signals are available at all instances in the signal processing chain. In this paper, all calculations are based on the raw (envelope-detected) image data, while all image displays show the logarithmic images.

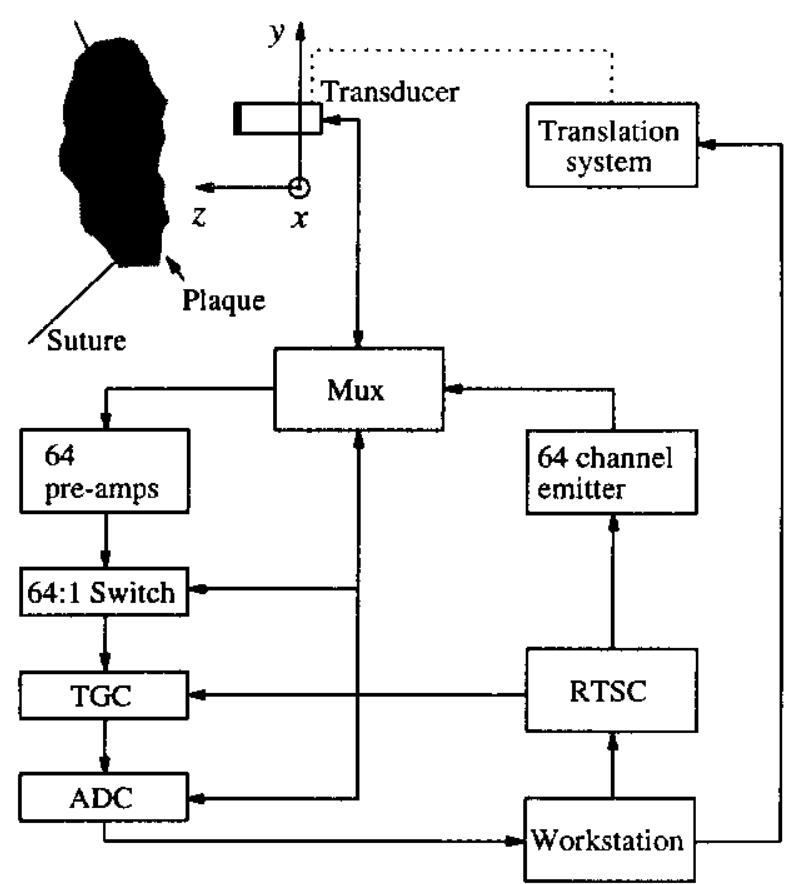

Figure 1 Block diagram of off-line ultrasound system used to scan formalin-fixed carotid atherosclerotic plaque in demineralized degassed water. The orientation of the coordinate system is used throughout this paper. 


\section{Scanning Procedure}

Atherosclerotic carotid plaques were removed by prophylactic endarterectomy and stored in formalin for several months. The plaques consist of the carotid artery intima (and some part of the media) as well as the plaque material that has been deposited on the intima. The plaques were mounted with sutures to an acrylic frame and submerged in demineralized degassed water at $20^{\circ} \mathrm{C}$ and scanned. The top part of Figure 1 provides a schematic illustration of the set-up. The distance between the cross-sectional scan planes was $0.5 \mathrm{~mm}$. A total of 48 image sets were recorded from two plaques. Each recorded image set consisted of seven single-angle images. An example of the corresponding compound image superimposed on a drawing showing transducer and fully compounded region ${ }^{[4]}$ is given in Figure 2. A transducer frequency of $7.5 \mathrm{MHz}$ was used, giving a wavelength $\lambda \cong 0.2 \mathrm{~mm}$ at sound speed, $c=1480 \mathrm{~m} / \mathrm{s}$.

\section{Image Generation and Outlining}

The raw (envelope-detected) image from a given scan plane and angle is denoted $I(z, x ; y, \theta)$ where $z$ and $x$ are the image coordinates, $y$ specifies the image plane and $\theta$ specifies the scan angle. The corresponding compound image is generated by averaging the raw single-angle images, however, it is only in the fully compounded region that all single-angle images contribute to this averaging. ${ }^{[4]}$ The multi-angle compound image is denoted $I_{\text {maci }}(z, x ; y)$.

The region containing plaque (ROI) in the ultrasound image - for a given scan plane - is identified with the following routine and saved as a "mask" image. Note that in a given scan plane, this region is the same for all

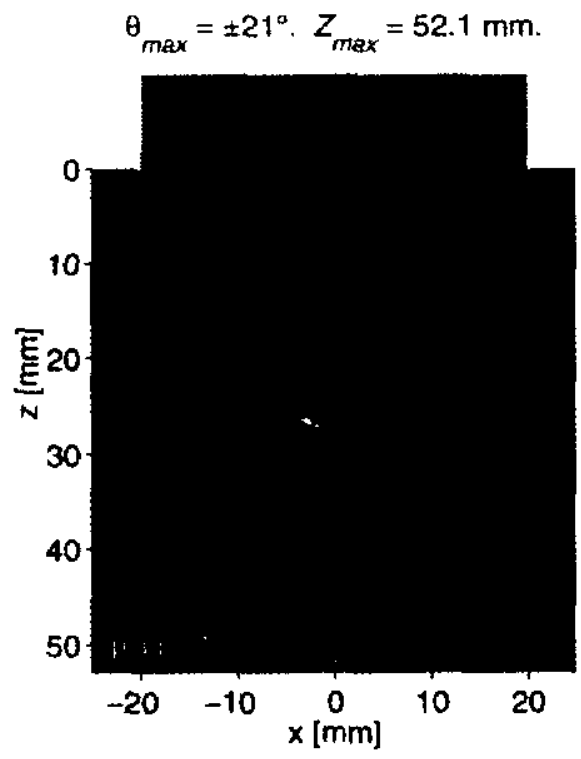

Figure 2 Cross-sectional view of transducer and fully compounded region with the corresponding ultrasound image superimposed. single-angle images and the compound image. The raw image contains values between 0 and some positive number. Initially, the mask image contains the values -1 .

1) A possible residual lumen is outlined manually from the compound image as this image is the best suited for this purpose..$^{[4]}$ The pixel value inside this region is set to 0 in the mask image.

2) A smooth outline slightly larger than the plaque is then drawn manually on the compound image, in order to effectively remove possible dual reflections, reflections from sutures, etc. Any pixel outside this region is set to 0 in the mask image.

3) An automatic threshold detection is then applied to the single-angle images, such that if a pixel is above the threshold in just one of the single-angle images, this pixel is identified as "plaque" pixel and the corresponding pixel in the mask is set to unity.

4) After this, the mask image will still contain some unresolved pixels (with value -1 ) in a band along the circumferential of the plaque region and possible some in anechoic regions inside the plaque. The first pixels are resolved by expanding the exterior background region (with value 0 ) in such a way that no pixel with value 0 is neighbor to a pixel with value -1 . Possible remaining pixels with value -1 will then correspond to anechoic regions. These are simply set to 1 .

The plaque region identified this way contained the entire plaque. In order to remove the part of the image that contained echoes from the interface between water and intima (which have a somewhat arbitrary level), the plaque region was shrunk by approximately the size of the vertical point spread function, which was approximately $\Delta z_{12 \mathrm{~dB}}=5 \lambda$. This corresponded to a band 20 pixels wide in the image. An example of two sets of outlines is given in Figure 3.

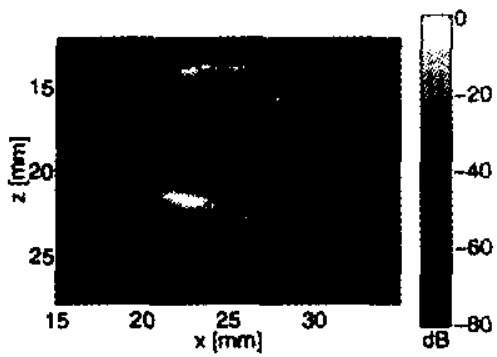

Figure 3 Example of ultrasound compound image of plaque cross-section with shrunken outline superimposed.

\section{Calculation of Mean Echogenicity}

The echogenicity of the plaque in each single-angle image for each scan plane was expressed by calculating the mean amplitude level, MAL, in the identified plaque region of the image as follows: 


$$
\begin{aligned}
& \operatorname{MAL}(y, \theta) \\
= & \frac{1}{N_{\mathrm{ROI}}(y)} \sum_{z} \sum_{x} l(z, x ; y, \theta) \times \operatorname{ROI}(z, x ; y)
\end{aligned}
$$

where $\operatorname{ROI}(z, x ; y)$ is the mask with values 0 and 1 , that specifies the region of interest (found by the method described in Section 4) and $N_{\mathrm{ROr}}(y)$ is the number of pixels inside this mask,

$$
N_{\mathrm{ROI}}(y)=\sum_{z} \sum_{x} \operatorname{ROI}(z, x ; y)
$$

\section{6 . Angular sensitivity of Ultrasound System}

For the comparison done in this paper, it is important to ensure that the angular sensitivity of the ultrasound system is constant as a function of angle. There are several factors that determine this angular sensitivity for the plaques scanned in this study:

1) The compensation that the scanner automatically applies to the raw received signals to compensate for the varying angular sensitivity of the individual transducer elements.

2) The shape and level of the TGC curve relative to the actual (unknown) loss in the plaque.

3) The overcompensation by the TGC curve, when the ultrasound travels in water and not plaque.

The effect of the first two items cannot be easily distinguished, while the third overcompensation could be removed by re-adjusting the TGC curve for each scanline, by carefully calculating path length from a plot like the one in Figure 2. However, as the cross-sectional shapes of the plaques were rather constant for the two plaques scanned in this investigation, such an approach might only yield a slight improvement probably smaller than the inaccuracies due to 1 ) and 2).

In order to obtain an estimate of the angular sensitivity of the ultrasound system in the present application, a compensation based on the $\operatorname{MAL}(y, \theta)$ was used. Specifically, the variation along the $y$-dimension in the MAL is due (mainly) to changes in plaque properties (e.g., plaque material, angle-dependence due to curvature in the $y$-direction) while the variation along the $\theta$-dimension is due to tissue induced angle-dependence in the scan plane and the (constant) undesired angular sensitivity. Assuming that the tissue induced angle-dependence in the scan plane varied randomly over the 48 image planes, the angular sensitivity was estimated by averaging over all 48 image planes. This was done the following way: For a given image plane, e.g., $y_{1}$, a parabola was fitted to the seven values of the MAL $\left(y_{1}, \theta\right)$. This gave 48 parabolas of different magnitudes and location, due to the variation of the MAL in the $y$-direction. A normalization factor was calculated from each parabola as the mean of the parabola values at $\pm 21^{\circ}$. Each row in the $\operatorname{MAL}(y, \theta)$ was then divided by the corresponding normalization factor before

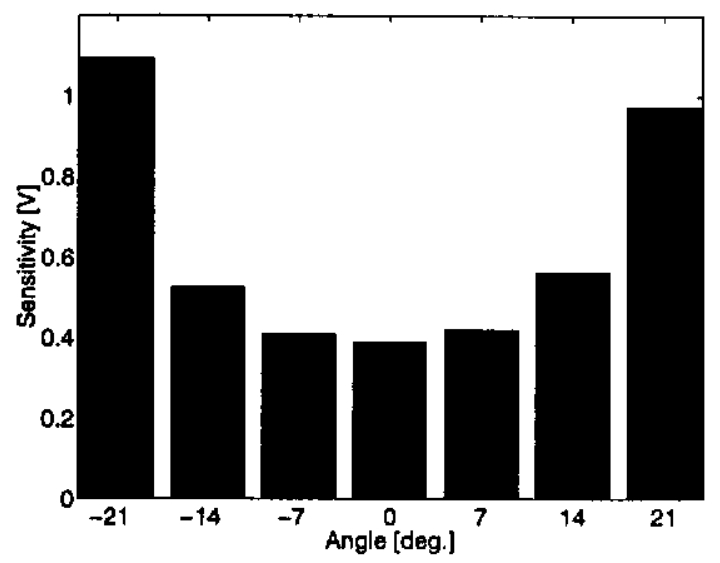

Figure 4 Estimate of the angular sensitivity of the ultrasound system when scanning the plaques used in this study.

averaging over the $y$-dimension was done. The result is shown in Figure 4.

Subsequently, the MAL could be compensated for the sensitivity function in Figure 4, by dividing each row of the MAL matrix with the function in Figure 4. The result of this compensation is given in Figure 5.

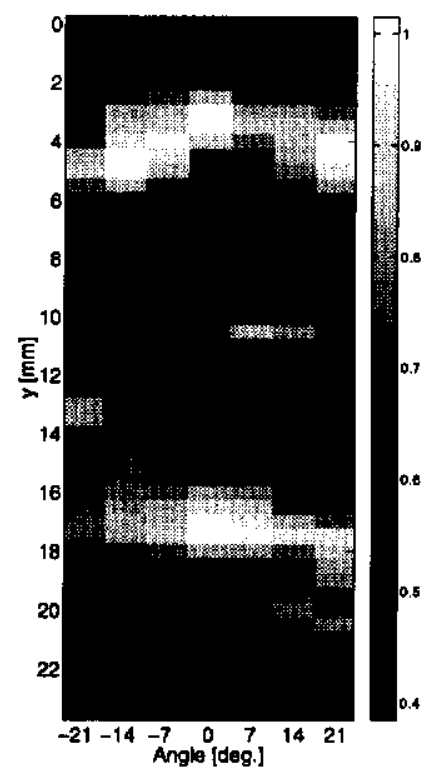

Figure 5 The compensated MAL, $\operatorname{MAL}_{\text {comp }}(y, \theta)$, showing a measure of the variation of the mean echogenicity from scan plane to scan plane and from angle to angle.

\section{Results}

In order to investigate the variation of the $\mathrm{MAL}_{\text {comp }}$ over scan planes and angles, the following parameters were calculated. The variation of the $\mathrm{MAL}_{\text {comp }}$ over scan plane was for each angle: 


$$
\sigma_{y}(\theta)=\operatorname{std}_{y}\left\{\operatorname{MAL}_{\text {comp }}(y, \theta)\right\}
$$

The variation of $\mathrm{MAL}_{\text {comp }}$ over angle was calculated as the standard deviation over angle as follows:

$$
\sigma_{\theta}(y)=\operatorname{std}_{\theta}\left\{\operatorname{MAL}_{\text {comp }}(y, \theta)\right\}
$$

$\sigma_{y}(\theta)$ and $\sigma_{\theta}=$ mean $\left\{\sigma_{\theta}(y)\right\}$ are plotted in Figure 6 for the two outlines described in Section 4. Furthermore, mean $\left\{\sigma_{\theta}(y)\right\} \pm$ std $\left\{\sigma_{\theta}(y)\right\} \cong 0.07 \pm 0.04 \mathrm{~V}$ for both outlines.

Similar figures were finally calculated from the $\mathrm{MAL}_{\text {comp }}$ with average over all angles:

$$
\sigma_{\text {maci }}(y)=\underset{\theta}{\operatorname{mean}}\left\{\operatorname{MAL}_{c o m p}(y, \theta)\right\}
$$

This gave $\operatorname{std}\left\{\sigma_{\text {maci }}(y)\right\}=0.135 \mathrm{~V}$.

\section{Discussion}

The two curves for $\sigma_{y}(\theta)$ in Figure 6 are nearly identical. Likewise for the two values of $\sigma_{\theta}$. This indicates that the echo signal from the water/plaque interface is not dramatically different from the echos originating from the internal part of the plaque.

The value of $\sigma_{y}(\theta)$ - for a given $\theta$ - indicates how the changes in plaque properties appear as changes in the MAL. The value of $\sigma_{\theta}$ indicates the typical variation over angle, for the same plaque region. Because $\sigma_{y}(\theta)>\sigma_{\theta}$ (approximately by a factor of two), it seems that the angular variation in the MAL is not so strong that it completely masks the variation in MAL due to plaque properties.

Eventually, because $\operatorname{std}\left\{\sigma_{\text {maci }}(y)\right\}>\sigma_{\theta}$ as well, this indicates that the variation in plaque properties is also reflected in the compound image (when generated based on compensated single-angle images).

There are a number of error sources in this investigation, that should be kept in mind:

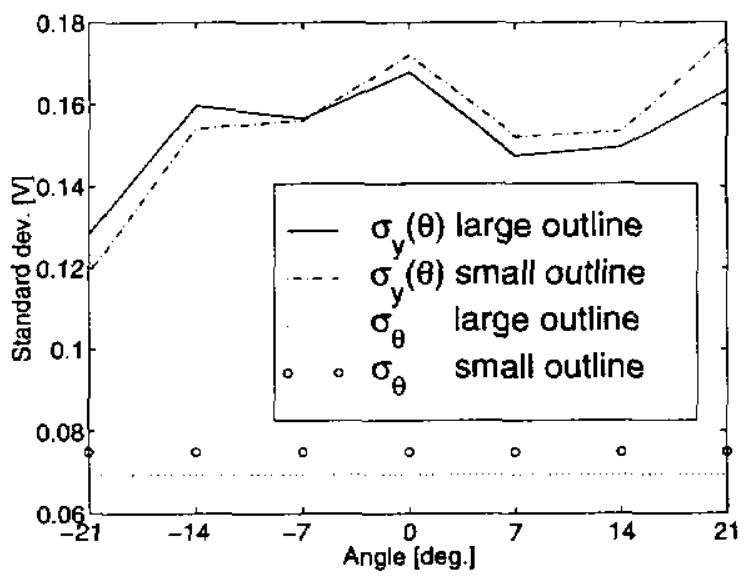

Figure 6 Standard deviations over scan planes and angles for the two different outline sets described in Section 4. a) Even though the echo from the interface between water and plaque was removed, the variation in attenuation in plaque was not known and therefore not compensated for.

b) The outlined plaque region could have an incorrect size. In future investigations, this should be verifyed, by measuring the physical area of the same plaque cross-section, for example, prior to a histological analysis.

Eventually it should be noted, that it is unknown to which degree the conclusions arrived at in this in vitro investigation can be extrapolated to the in vivo situation, where temperature and physical properties of the plaque and surroundings are different.

\section{Conclusions}

Formalin-fixed atherosclerotic plaques removed by surgery was scanned with $7.5 \mathrm{MHz}$ ultrasound from seven different angles, by use of a 192-element linear array transducer connected to a 64-channel experimental ultrasound system. Forty-eight images were recorded, from where the mean echogenicity was calculated inside the plaque region on each image. The results showed that the variation in mean echogenicity over scan plane was larger than the variation over angle. This in vitro result thus indicates that the variation in mean echogenicity due to changes in plaque properties is larger than the variation due to change in insonification angle, when the insonification angle range is below $42^{\circ}$.

\section{Acknowledgements}

CADUS is supported by the Danish Technical and Medical Research Councils. The authors gratefully acknowledge the help from M.D. M.-L. M. Grønholdt for supplying the biological specimens and by instrument maker K. Martinsen for making fixtures.

\section{References}

[1] European Carotid Plaque Study Group (ECPSG): Carotid artery plaque composition - relationship to clinical presentation and ultrasound B-mode imaging. Eur. J. Vasc. Endovasc. Surg., Vol. 10, pp. 23-30, 1995.

[2] Feeley TM, Leen EJ, Colgan M-P, Moore DJ, Hourihane DO'B, Shanik GD: Histologic characteristics of carotid artery plaque. J. Vasc. Surg., Vol. 13, No. 5, pp. 719-724, 1991.

[3] O'Donnell TF, Erdoes L, Mackey WC, McCullough J, Shepard A, Heggerick P, Isner J, Callow AD: Correlation of B-mode ultrasound imaging and arteriography with pathologic findings at carotid endarterectomy. Arch Surg., Vol. 120, pp. 443-449, 1985.

[4] Jespersen SK, Wilhjelm JE \& Sillesen H: Ultrasound Spatial Compound Scanner for Improved Visualization in Vascular Imaging. 1998 IEEE Int. Ultrasonic Symp., Sendai, Miyagi, Japan. 1998. (This proceeding.) 